\title{
A potential prognostic prediction model of colon adenocarcinoma with recurrence based on prognostic IncRNA signatures
}

Lipeng Jin, Chenyao Li, Tao Liu and Lei Wang*

\begin{abstract}
Background: Colon adenocarcinoma (COAD) is one of the common gastrointestinal malignant diseases, with high mortality rate and poor prognosis due to delayed diagnosis. This study aimed to construct a prognostic prediction model for patients with colon adenocarcinoma (COAD) recurrence.

Methods: Differently expressed RNAs (DERs) between recurrence and non-recurrence COAD samples were identified based on expression profile data from the NCBI Gene Expression Omnibus (GEO) repository and The Cancer Genome Atlas (TCGA) database. Then, recurrent COAD discriminating classifier was established using SMVRFE algorithm, and receiver operating characteristic curve was used to assess the predictive power of classifier. Furthermore, the prognostic prediction model was constructed based on univariate and multivariate Cox regression analysis, and Kaplan-Meier survival curve analysis was used to estimate this model. Furthermore, the co-expression network of DEIncRNAs and DEmRNAs was constructed followed by GO and KEGG pathway enrichment analysis.
\end{abstract}

Results: A total of 54 optimized signature DEIncRNAs were screened and SMV classifier was constructed, which presented a high accuracy to distinguish recurrence and non-recurrence COAD samples. Furthermore, six independent prognostic IncRNAs signatures (LINC00852, ZNF667-AS1, FOXP1-IT1, LINC01560, TAF1A-AS1, and LINC00174) in COAD patients with recurrence were screened, and the prognostic prediction model for recurrent COAD was constructed, which possessed a relative satisfying predicted ability both in the training dataset and validation dataset. Furthermore, the DEmRNAs in the coexpression network were mainly enriched in glycan biosynthesis, cardiac muscle contraction, and colorectal cancer.

Conclusions: Our study revealed that six IncRNA signatures acted as an independent prognostic biomarker for patients with COAD recurrence.

Keywords: Colon adenocarcinoma, Recurrence, Differentially expressed genes, Prognosis

\section{Introduction}

As one of the most common gastrointestinal malignant diseases, colon adenocarcinoma (COAD) is the worldwide leading cause of mortality [1]. Currently, the standard therapeutic method for COAD is the combination of surgery and adjuvant chemotherapy or radiation therapy

\footnotetext{
*Correspondence: leiwang_1967@163.com

Department of Colorectal \& Anal Surgery, First Hospital Bethune of Jilin University, No. 71, Xinmin Street, Chaoyang District, Changchun, Jilin 130000, China
}

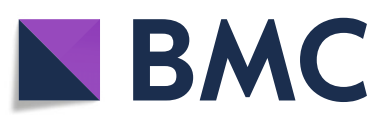

[2]. Additionally, the early diagnosis for primary or recurrent COAD is also a critical factor for improving the prognosis of patients [3]. Unfortunately, despite substantial advances in early diagnosis and treatments, poor survival, high recurrence, and unsatisfactory prognosis remain an issue due to delayed diagnosis and adverse drug effects [2, 4]. Therefore, identification of novel diagnostic, prognostic biomarkers and therapeutic targets, as well as investigation of the underlying molecular mechanism of COAD, is required.

(c) The Author(s). 2020 Open Access This article is licensed under a Creative Commons Attribution 4.0 International License, which permits use, sharing, adaptation, distribution and reproduction in any medium or format, as long as you give appropriate credit to the original author(s) and the source, provide a link to the Creative Commons licence, and indicate if changes were made. The images or other third party material in this article are included in the article's Creative Commons licence, unless indicated otherwise in a credit line to the material. If material is not included in the article's Creative Commons licence and your intended use is not permitted by statutory regulation or exceeds the permitted use, you will need to obtain permission directly from the copyright holder. To view a copy of this licence, visit http://creativecommons.org/licenses/by/4.0/ The Creative Commons Public Domain Dedication waiver (http://creativecommons.org/publicdomain/zero/1.0/) applies to the data made available in this article, unless otherwise stated in a credit line to the data. 
Ideal diagnostic and prognostic biomarkers should be strongly associated with the prognosis of patients and easy to detect [5]. Encouragingly, overwhelming evidence has demonstrated that the regulatory roles of noncoding RNAs such as long non-coding RNAs (lncRNAs) are predominately correlated with the development and progression of a wide variety of cancers [6]. LncRNAs are arbitrarily defined as noncoding RNA with the length > 200 nucleotides, and have several possible functions, including miRNA sponges, regulating gene transcription and splicing, and forming RNAprotein complexes [7]. It is now well appreciated that lncRNAs participate in disease progression by regulating various key cell biological processes such as cell proliferation, differentiation, apoptosis, migration, and invasion [8]. To date, lncRNAs have been revealed abnormal expression in cancers, and some of which are served as oncogenes or tumor suppressors [8]. Furthermore, accumulating studies have shown that lncRNAs are potentially identified as novel diagnostic, prognostic, and metastasis predictive biomarkers in various cancers [9-11]. Recently, several lncRNA profiling has identified several colorectal cancer-specific lncRNAs, and the following experiments have demonstrated that lncRNAs such as PCAT-1, RP11-462C24.1, HOTAIR, and MALAT1 are candidate diagnostic biomarkers [12-14]. However, few studies have investigated lncRNAs as the prognostic biomarkers for recurrent COAD.

In the current study, lncRNAs related to COAD recurrence were screened based on expression profile data from the National Center for Biotechnology Information (NCBI) GEO repository and The Cancer Genome Atlas (TCGA). Next, a recurrent COAD discriminating classifier and a prognostic prediction model were constructed using the bioinformatics methods. Moreover, coexpression network and pathways were analyzed. According to this, we aimed to explore a useful prognostic prediction model for recurrent COAD and provide some useful insights in improving the prognosis of recurrent COAD patients.

\section{Materials and methods}

\section{Data extraction and preprocessing}

The gene expression datasets were preliminarily extracted from the NCBI GEO repository (https://www. ncbi.nlm.nih.gov/geo/) using search words of "colon adenocarcinoma and Homo sapiens". Then, the eligible dataset were selected in this study according to the following criteria: (1) the samples in datasets were solid tissues of COAD patients; (2) the total number of COAD samples was not less than 500; and (3) the datasets contained recurrence and prognosis information of samples. Eventually, GSE39582 was obtained and utilized as training dataset. This dataset was generated from GPL570 Affymetrix Human Genome U133 Plus 2.0 Array platform and contained 585 COAD samples and 574 samples had recurrence information [15].

Meanwhile, the RNA sequencing data and corresponding clinical information of COAD patients were downloaded from TCGA (https://gdc-portal.nci.nih.gov/). This dataset was obtained from the platform of Illumina HiSeq 2000 RNA Sequencing and contained 512 COAD samples. After the RNA sequencing data was matched with clinical information, a total of 310 samples containing recurrence and prognosis information were obtained, which was utilized as validation dataset. The clinical characteristics of COAD patients in the training and validation datasets are shown in Table 1.

\section{Screening of differentially expressed RNAs (DERs)}

Firstly, all mRNAs and lncRNAs in training and validation dataset were annotated based on the HUGO Gene Nomenclature Committee (HGNC, http://www.genenames.org/) database [16], consisting of annotated 19, 198 protein coding genes and 4120 lncRNAs. Then, the overlapping mRNAs and lncRNAs were obtained between these two datasets. All COAD samples in the training dataset were divided into recurrence and nonrecurrence groups. The limma package (version 3.34.7, https://bioconductor.org/packages/release/bioc/html/ limma.html) [17] in R 3.4.1 was utilized to screen DERs (including mRNA and lncRNA) between recurrence and non-recurrence samples with the thresholds of false discovery rate $<0.05$ and $\mid \log _{2}$ fold change $\mid>0.263$. Furthermore, bidirectional hierarchical clustering based on centered Pearson correlation algorithm [18] was performed by pheatmap (Version 1.0.8, https://cran.r-project.org/web/packages/pheatmap/index.html) [19] in $\mathrm{R}$ 3.4.1 according to the expression values of DERs in the training dataset.

\section{Screening of signature IncRNAs}

The e1071 (Version 1.7-1, https://cran.r-project.org/web/ packages/e1071) [20] and caret package (Version 6.0-76, https://cran.r-project.org/web/packages/caret) [21] in R was used to identify optimized signature lncRNAs based on recursive feature elimination (RFE) algorithm. Next, the SVM-based classifier was built to predict COAD recurrence based on signature lncRNAs. In addition, the performance of the classifier was evaluated in the training dataset and validation dataset, respectively. The area under curve (AUC) index was calculated to evaluate the predictive power of the classifier based on receiver operating characteristic (ROC) curve analysis using pROC (Version 1.15.0, https://cran.r-project.org/web/packages/pROC/index.html) [22] in R. The corresponding parameters, including sensitivity (Sen), specificity (Spe), positive prediction value (PPV), and negative prediction value (NPV), were also calculated using pROC. 
Table 1 Clinical characteristics of the colon adenocarcinoma samples in the training and validation datasets

\begin{tabular}{lll}
\hline Clinical characteristics & Training dataset $(\mathrm{N}=574)$ & Validation dataset $(\mathrm{N}=310)$ \\
\hline Age at pathologic diagnosis (years, mean $\pm \mathrm{sd})$ & $66.89 \pm 13.22$ & $65.73 \pm 12.71$ \\
Gender (male/female) & $317 / 257$ & $169 / 141$ \\
Pathologic stage (1/2/3/4) & $41 / 267 / 206 / 60$ & $51 / 118 / 88 / 43 / 10$ \\
Pathological T (1/2/3/4/-) & $13 / 47 / 373 / 118 / 23$ & $8 / 55 / 212 / 34 / 1$ \\
Pathological N (0/1/2/3/-) & $309 / 134 / 99 / 6 / 26$ & $180 / 77 / 53 / 0 / 0$ \\
Pathological M (0/1/-) & $491 / 61 / 22$ & $226 / 43 / 41$ \\
Tumor location (distal/proximal) & $348 / 226$ & - \\
Chemotherapy (yes/no) & $239 / 319 / 16$ & - \\
Tumor recurrence (yes/no) & $179 / 375$ & $66 / 244$ \\
Recurrence free survival time (months, mean $\pm \mathrm{sd})$ & $49.79 \pm 40.76$ & $29.66 \pm 25.46$
\end{tabular}

\section{Constructions and verification of prognostic prediction model}

The univariate cox regression analysis for the lncRNAs used for SVM classifier construction was carried out using survival package (Version 2.41.1, http://bioconductor.org/packages/survivalr/) [23] in R3.4.1 with the threshold of $\log$-rank $P$ value $<0.05$. Then, independent prognostic lncRNAs were further screened by multivariate cox regression analysis using survival package (Version 2.41.1) [23]. Afterwards, the risk score (RS) prognostic prediction model was constructed based on expression levels of independent prognostic lncRNAs and their regression coefficients estimated from the multivariate Cox regression model as follows: $\mathrm{RS}=$ $\Sigma \beta_{\text {lncRNA }} \times$ Exp $_{\text {IncRNA }}$. The $\beta_{\text {lncRNA }}$ represented the independent prognostic coefficient and ExplncRNA was defined as the expression value of corresponding lncRNA. According to the median value of RS, all samples in the training dataset were divided into high-risk and low-risk groups. The Kaplan-Meier (K-M) survival curve analysis was performed to evaluate survival difference between high- and low-risk group using survival package (version 2.41.1) in $R$ 3.4.1. Moreover, the prognostic significance of RS was also assessed by including C-index [24], Brier score [25], and log-rank $P$ value of cox- $\mathrm{PH}$ regression [26]. Similarly, a RS model was also established in validation set. Accordingly, K-M curves were constructed to analyze two risk groups and COAD survival. C-index, Brier score, and log-rank $P$ value $C$-index were used to evaluate the predictive accuracy of RS.

\section{Construction of co-expression network and functional analysis}

The expression levels of the signature lncRNAs and differentially expressed mRNAs were extracted from COAD samples in the training dataset. Next, co-expression network was constructed based on Pearson correlation coefficient (PCC) of the prognostic lncRNAs and DE mRNAs using cor.test function (https://stat.ethz.ch/R-manual/R- devel/library/stats/html/cor.test.html) [27] in R3.4.1 and then visualized by Cytoscape (Version 3.6.1, https://cytoscape.org/). Besides, Gene Ontology (GO) functional annotation associated with biological process analysis as well as Kyoto Encyclopedia of Genes and Genomes (KEGG) pathway enrichment analysis of mRNAs in co-expression network were performed based on Database for Annotation, Visualization, and Integrated Discovery (DAVID) program (v 6.8, https://david.ncifcrf.gov/) [28, 29] with the threshold of $P$ value $<0.05$.

\section{Results}

DERs screening between recurrence and non-recurrence COAD samples

This study was conducted as indicated in Fig. 1. After annotation, there were 13834 mRNAs and 827 lncRNAs between training and validation dataset. Subsequently, a total of 1002 DERs were identified between recurrence $(n=179)$ and non-recurrence $(n=375)$ COAD samples based on the selective criteria, including 939 DE mRNAs (475 downregulated and 464 upregulated mRNAs) and 63 DE lncRNAs (13 downregulated and 50 upregulated lncRNAs) (Fig. 2a). The bidirectional hierarchical clustering analysis indicated that these DERs could significantly distinguish recurrent and non-recurrent COAD samples (Fig. 2b).

\section{Signature IncRNA screening}

The SVM-RFE algorithm was used to identify the most optimized lncRNA signatures. We found that there were 54 optimized lncRNA signatures when the accuracy was the highest value of 0.879 (Fig. 3a). Then, a SMV classifier was established based on the 54 optimized lncRNAs to differentiate recurrent COAD samples from non-recurrent COAD samples. ROC curve analysis revealed that this SVM classifier exhibited a good discriminatory power for patients with or without COAD recurrence in training dataset (AUC 0.989, Sen 0.911, Spe 0.987, PPV 0.970, and NPV 0.961; Fig. 3b). Similarly, a SVM-based classifier was also built in validation set and it had a high accuracy to distinguish recurrent and non- 


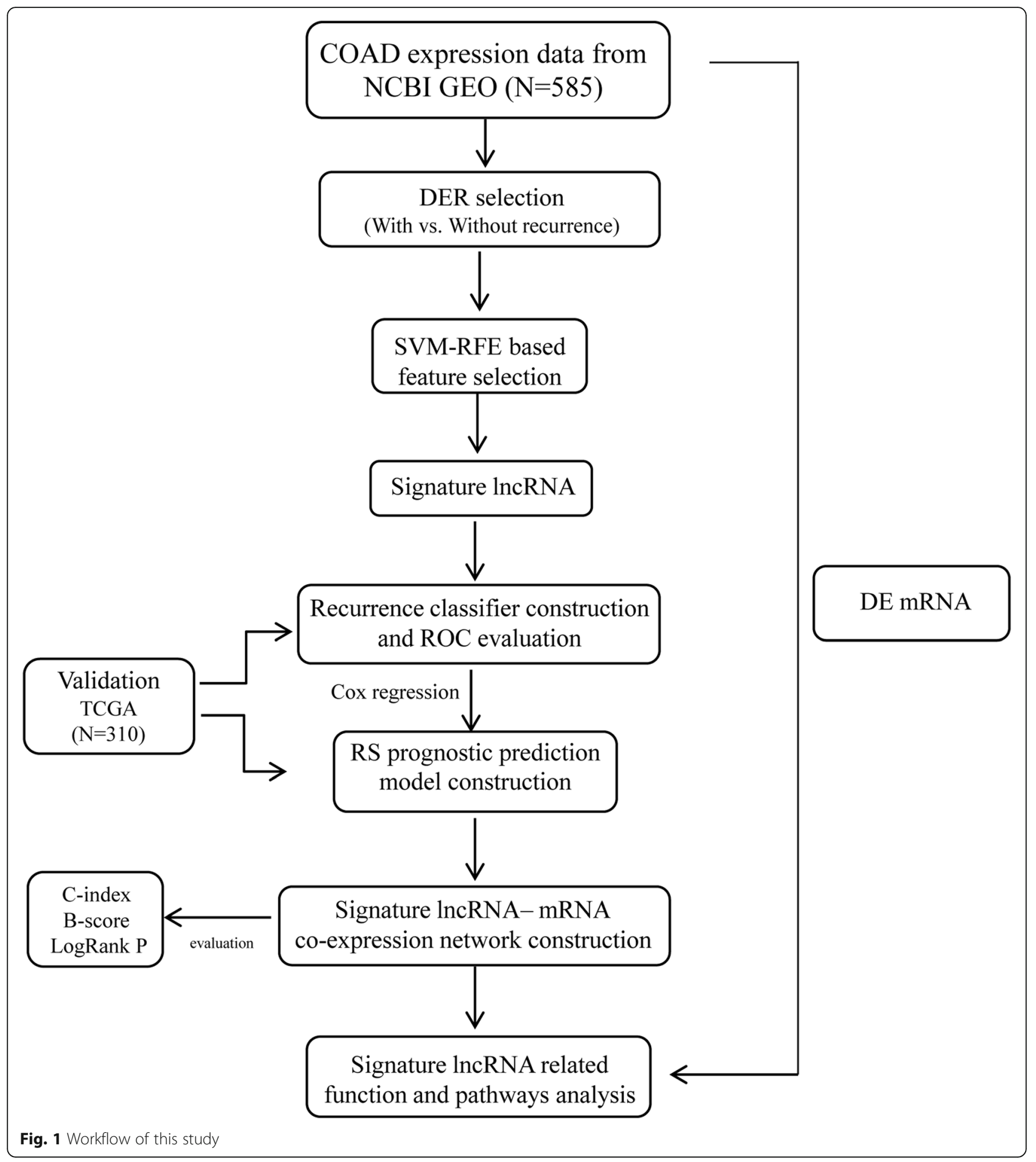

recurrent COAD samples (AUC 0.920, Sen 0.803, Spe 0.877, PPV 0.739, and NPV 0.930; Fig. 3b).

\section{Construction and verification of prognostic prediction} model

Based on univariate Cox regression analysis in the training dataset, a total of 39 DElncRNAs were significantly associated with the overall survival of patients with COAD recurrence. Then, six independent prognostic lncRNAs (LINC00852, ZNF667-AS1, FOXP1-IT1, LINC01560, TAF1A-AS1, and LINC00174) were obtained by multivariate Cox regression analysis (Table 2). Among them, two lncRNAs (LINC00852, FOXP1-IT1) with negative coefficients revealed that their higher 


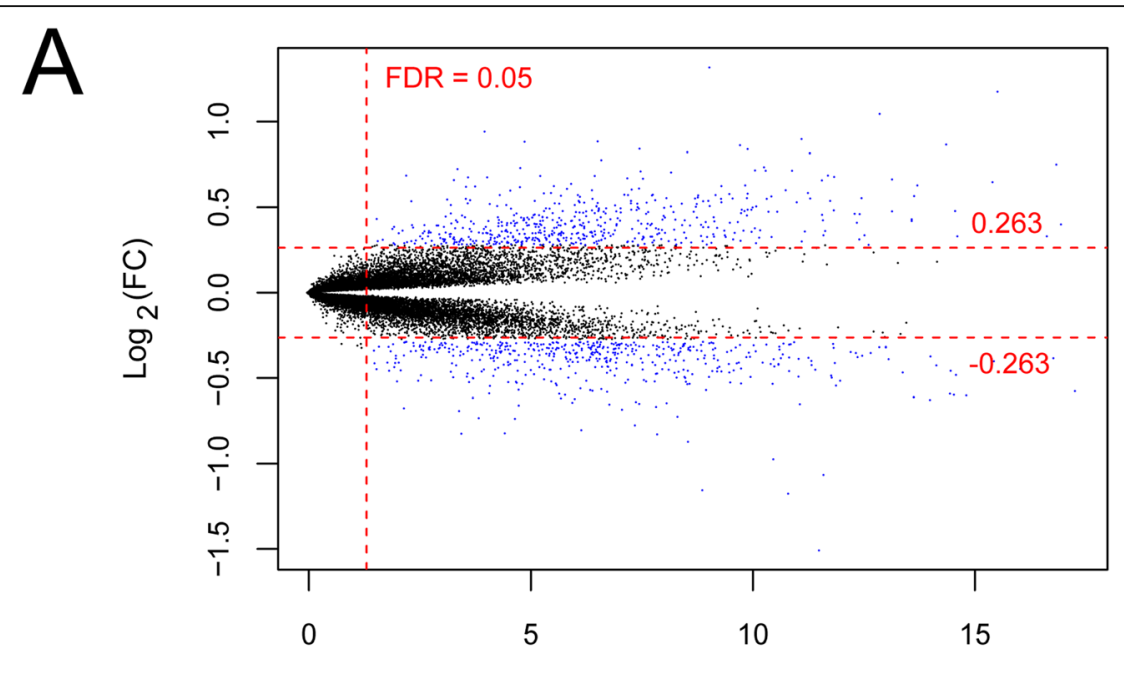

$(-1)^{\star} \log 10($ FDR)

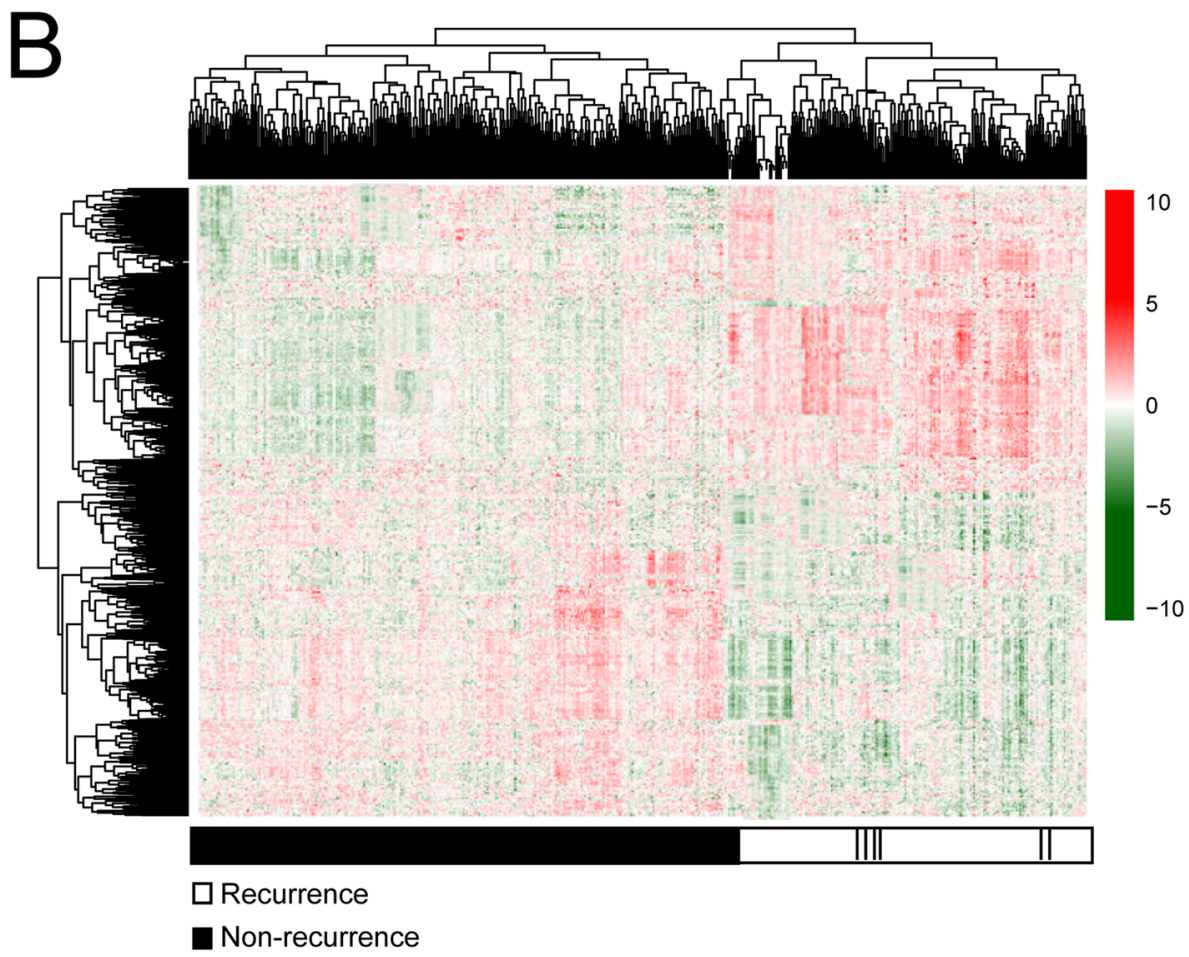

Fig. 2 Identification of differentially expressed RNAs (DERs). a Volcano map. Blue dots indicate DERs, the red horizontal dotted line represents false discovery rate $(F D R)<0.05$, the two red vertical dotted lines represent the $\mid \log$ fold change $(F C) \mid>0.263$. b A bidirectional hierarchical clustering map based on DERs. White and black sample bars represent recurrence and non-recurrence colon adenocarcinoma samples, respectively

expressions were correlated with longer survival, while the remaining lncRNAs (ZNF667-AS1, LINC01560, TAF1AAS1, and LINC00174) with positive coefficients indicated that their higher expression were associated with unfavorable survival outcomes. Next, the RS prognostic prediction model was constructed based on the coefficients of six independent prognostic lncRNAs and their expression levels in training dataset as follows: RS $=(-0.9109) \times$ $\operatorname{Exp}_{\text {LINC00852 }}+(0.4241) \times \operatorname{Exp}_{\text {ZNF667-AS1 }}+(-0.4840) \times$ Exp $_{\text {FOXP1-IT1 }}+(0.3945) \times$ Exp $_{\text {LINC01560 }}+(0.4466) \times$ Exp $_{\text {TAF1A-AS1 }}+(0.6742) \times$ Exp $_{\text {LINC00174. Subsequently, the }}$ RS of each patient was calculated and all patients were then divided into high-risk group $(n=268)$ and low-risk group ( $n=268)$ according to the median value of 

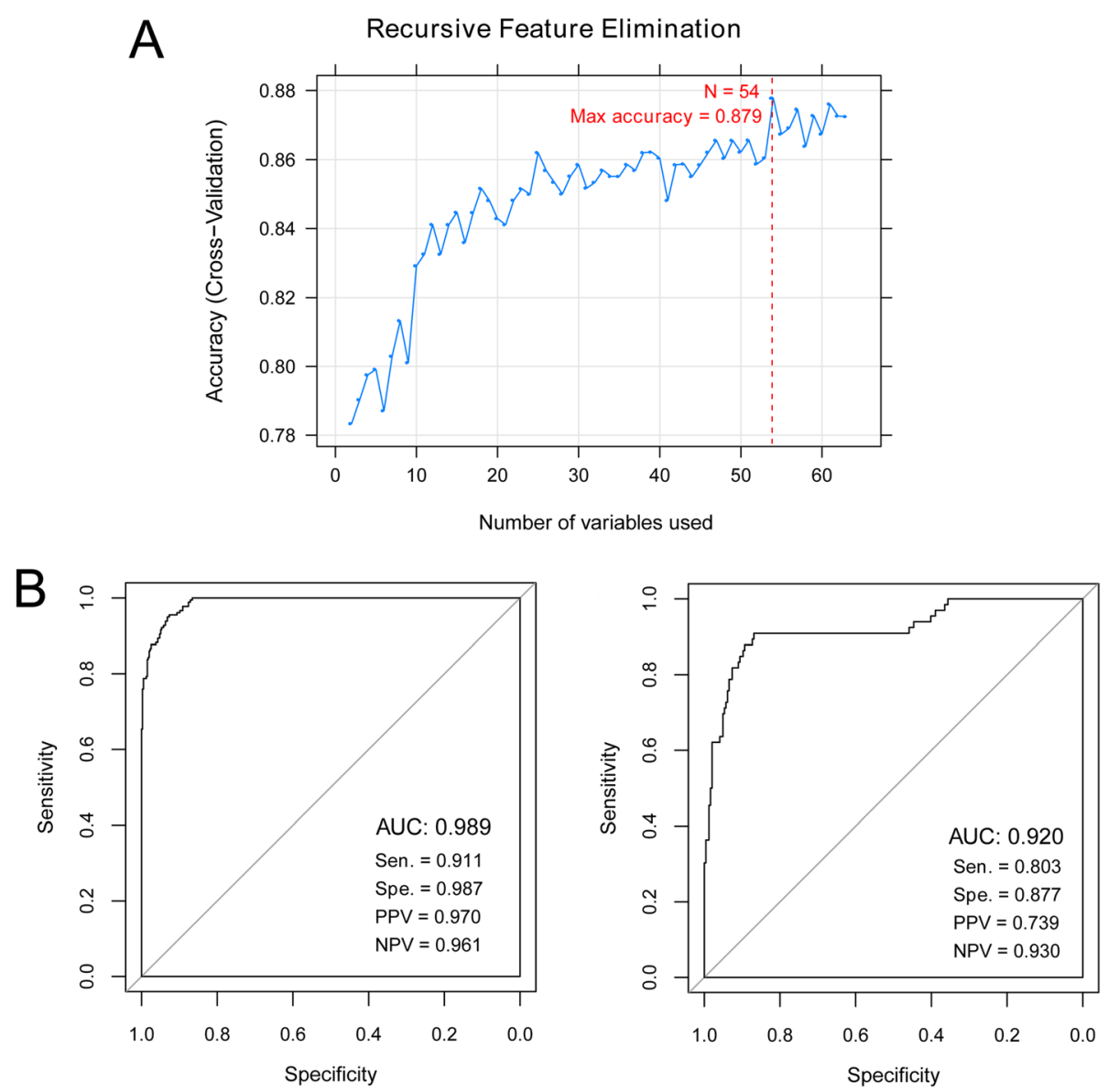

Fig. 3 Screening of signature IncRNAs and construction of recurrence colon adenocarcinoma (COAD) discriminating classifier. a Accuracy curve of the optimized signature IncRNAs using recursive feature elimination (RFE) algorithm. b Receiver operating characteristic (ROC) curve based on the support vector machine (SVM) classifier in the training dataset (left) and validation dataset (right)

RS (Table 3). Accordingly, the distribution of the RS and survival status of the GC patients as well as the expression levels of six prognostic lncRNA signatures were also obtained. As displayed in Fig. 4a, there were high expressions of risky lncRNAs (ZNF667-AS1, LINC01560, TAF1A-AS1, and LINC00174) in patients from the high-risk group. Conversely, those patients in the low-risk group tended to express high levels of protective lncRNAs (LINC00852, FOXP1-IT1). These findings were verified in the validation

Table 2 Six independent prognostic IncRNAs in colon adenocarcinoma patients with recurrence

\begin{tabular}{lllll}
\hline ID & Coef & P value & Hazard ratio & $95 \% \mathrm{Cl}$ \\
\hline LINC00852 & -0.9109 & $2.73 \mathrm{E}-03$ & 0.4022 & $0.2217-0.7296$ \\
ZNF667-AS1 & 0.4241 & $1.68 \mathrm{E}-02$ & 1.5282 & $1.0794-2.1638$ \\
FOXP1-IT1 & -0.4840 & $2.32 \mathrm{E}-02$ & 0.6163 & $0.4059-0.9359$ \\
LINC01560 & 0.3945 & $2.85 \mathrm{E}-02$ & 1.4836 & $1.0424-2.1115$ \\
TAF1A-AS1 & 0.4466 & $3.90 \mathrm{E}-02$ & 1.5630 & $1.0229-2.3884$ \\
LINC00174 & 0.6742 & $4.31 \mathrm{E}-02$ & 1.9624 & $1.0210-3.7718$ \\
\hline
\end{tabular}

dataset as shown in Fig. 4b. Additionally, the effects of six prognostic lncRNAs on survival outcomes of COAD patients in high- and low-risk group were also assessed by K-M analysis in training and validation sets. The results suggested that there was a lower survival probability for COAD patients in the high-risk group than that in the low-risk group (training dataset: a $\log$-rank $P$ value $=1.392 \mathrm{e}-09, \mathrm{HR}=2.878,95 \% \mathrm{CI}$ 2.012-4.118, C-index $=0.744$, and Brier score $=0.036$; validation dataset: a $\log$-rank $P$ value $=2.081 \mathrm{e}-02, \mathrm{HR}=1.786$, $95 \% \mathrm{CI} 1.085-2.940, \mathrm{C}$-index $=0.664$, and Brier score $=$ 0.063; Fig. 5).

\section{Construction of co-expression network and functional analysis}

The co-expression analysis between prognostic lncRNAs and DEmRNAs was performed. There were $198 \mathrm{DE}$ lncRNA-DE mRNA pairs among 6 lncRNAs, and 162 DE mRNAs (such as CXCL14, EPDR1, PMEPA1, HEPACAM, ST6GALNAC1, and SELENBP1) were obtained (Fig. 6a). Subsequently, the functional analyses of DEmRNAs in co-expression network were conducted 
Table 3 Independent prognostic factors of colon adenocarcinoma patient with recurrence by univariate and multivariate Cox regression analysis

\begin{tabular}{|c|c|c|c|c|c|c|}
\hline \multirow[t]{2}{*}{ Clinical characteristics } & \multicolumn{3}{|c|}{ Univariable Cox } & \multicolumn{3}{|c|}{ Multivariable Cox } \\
\hline & $\mathrm{HR}$ & $95 \% \mathrm{Cl}$ & P & $\mathrm{HR}$ & $95 \% \mathrm{Cl}$ & P \\
\hline Age at pathologic diagnosis (years, mean $\pm \mathrm{sd}$ ) & 1.011 & $0.997-1.023$ & $1.268 \mathrm{E}-01$ & - & - & - \\
\hline Gender (male/female) & 1.309 & $0.940-1.823$ & $1.096 \mathrm{E}-01$ & - & - & - \\
\hline Pathologic stage (1/2/3/4) & 1.866 & $1.475-2.360$ & $1.754 \mathrm{E}-07$ & 1.365 & $0.899-2.073$ & $1.439 \mathrm{E}-01$ \\
\hline Pathological T (1/2/3/4/-) & 1.969 & $1.443-2.686$ & $2.606 \mathrm{E}-05$ & 1.686 & $1.199-2.369$ & $2.630 \mathrm{E}-03$ \\
\hline Pathological N (0/1/2/3/-) & 1.691 & $1.387-2.059$ & $5.525 \mathrm{E}-07$ & 1.328 & $0.967-1.824$ & $7.921 \mathrm{E}-02$ \\
\hline Pathological M (0/1/-) & 1.77 & $0.861-3.639$ & $1.154 \mathrm{E}-01$ & - & - & - \\
\hline Tumor location (distal/proximal) & 0.346 & $0.602-1.181$ & $3.217 \mathrm{E}-01$ & - & - & - \\
\hline Chemotherapy (yes/no) & 1.647 & $1.190-2.277$ & $2.331 E-03$ & 0.998 & $0.669-1.490$ & $9.922 \mathrm{E}-01$ \\
\hline RS model status (high/low) & 2.878 & $2.012-4.118$ & $1.392 \mathrm{E}-09$ & 2.559 & $1.747-3.748$ & 1.410E-06 \\
\hline Tumor recurrence (yes/no) & - & - & - & - & - & - \\
\hline Recurrence free survival time (months, mean $\pm \mathrm{sd}$ ) & - & - & - & - & - & - \\
\hline
\end{tabular}

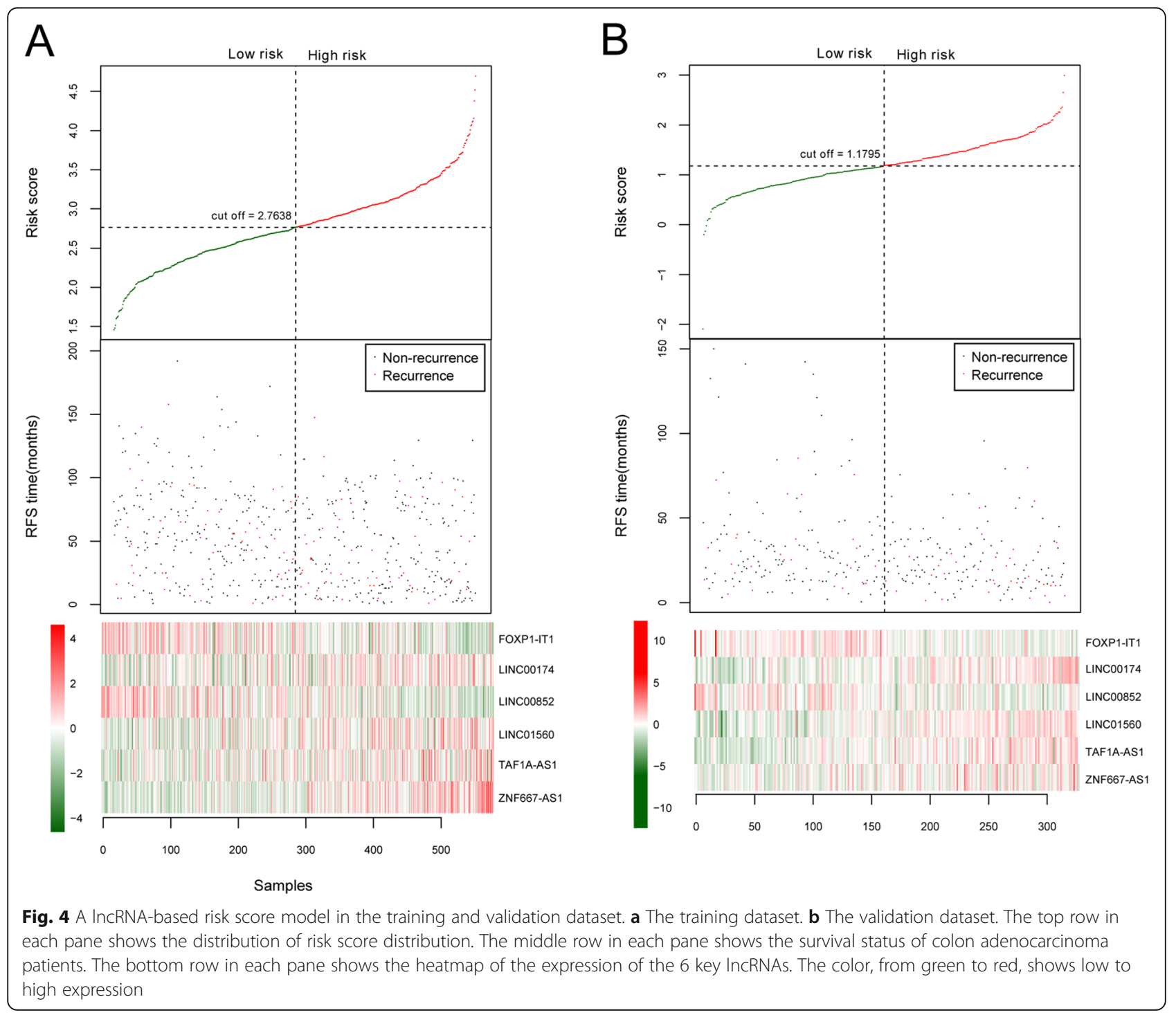




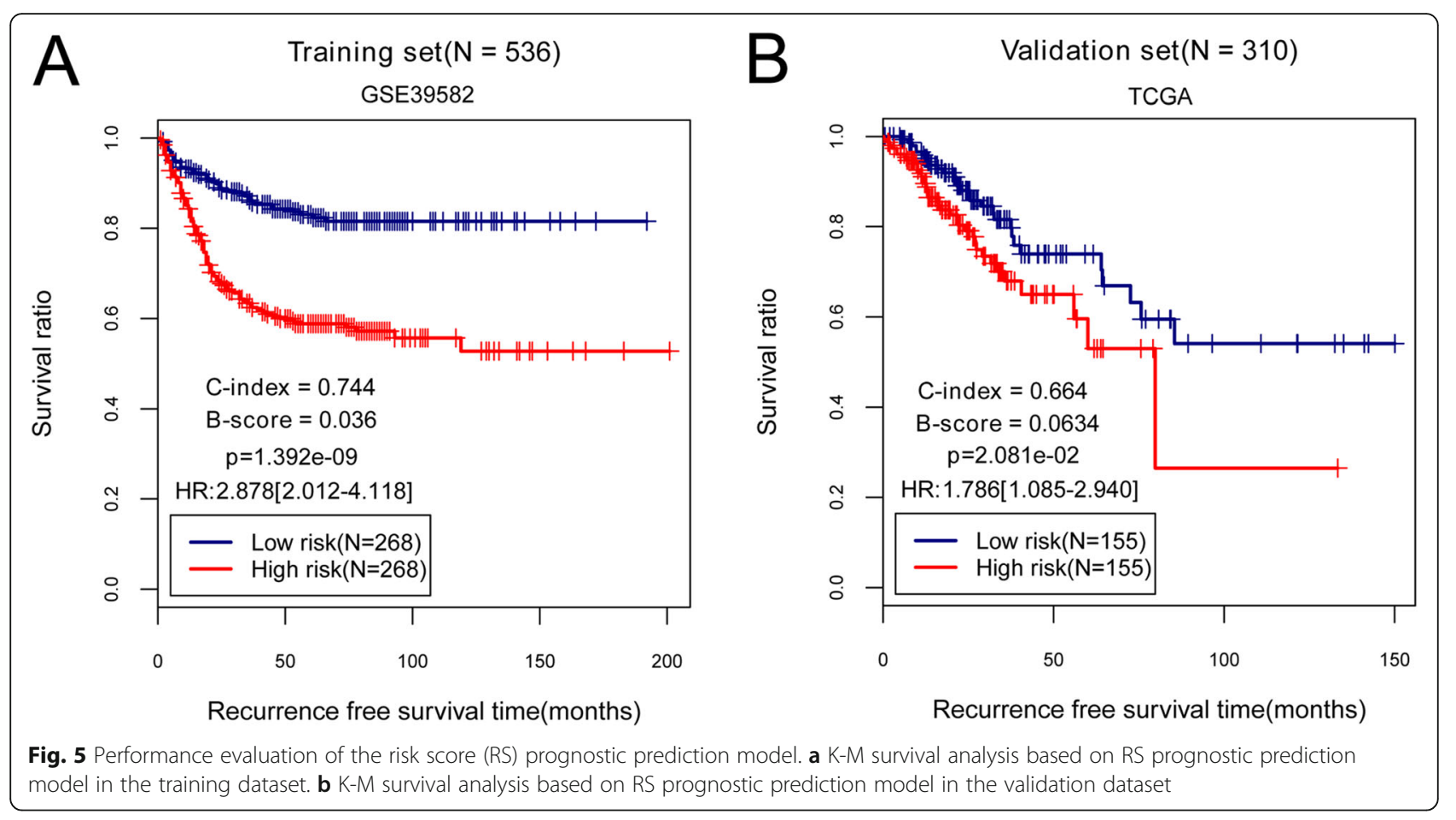

and the results revealed that these genes were significantly enriched in 17 GO-BP terms such as regulation of transcription, and 5 KEGG pathways, including glycan biosynthesis, cardiac muscle contraction, MAPK signaling pathway, colorectal cancer, and apoptosis (Fig. 6b).

\section{Discussion}

In this study, 939 DEmRNAs and 63DElncRNAs were identified between recurrent and non-recurrent COAD. Then, 54 optimized signature DElncRNAs were screened and subjected to a SMV classifier construction. Furthermore, 6 independent prognostic lncRNAs signatures (LINC00852, ZNF667-AS1, FOXP1-IT1, LINC01560, TAF1A-AS1, and LINC00174) were screened by univariate and multivariate Cox regression analysis, and the RS prognostic prediction model for recurrent COAD was constructed and showed a high predictive value for COAD samples both in the training dataset and validation dataset. Furthermore, the co-expression network revealed that LINC01560 interacted with CXCL14 and EPDR1 while FOXP1-IT1 was co-expressed with HEPACAM and ST6GALNAC1.

The mining of accumulating gene expression profile data in a variety of diseases have been enhanced with the rapid advances in high-throughput sequencing and bioinformatics technologies [30]. This study integrated the eligible expression profile data of COAD samples with and without recurrence from NCBI GEO and screened 63 DElncRNAs related to COAD recurrence. As a powerful classification tool, SVM is widely applied into cancer genomic subtyping or classification [31]. Due to the classification feature of SVM based on the large amounts of genomic data, SVM has been used to distinguish disease subtype [32], as well as discovery novel biomarkers or drug targets [31]. Similarly, this study also constructed a recurrent COAD discriminating classifier using SMV, which presented a good discriminatory capability for recurrent and non-recurrent COAD. Furthermore, the RS prognostic prediction model for recurrent COAD was constructed based on univariate and multivariate Cox regression analysis. Computationally, univariate and multivariate Cox regression were the most common method to construct the prognostic models and screen prognostic factors [33].

Co-expression network revealed that LINC01560 was co-expressed with CXCL14 and EPDR1, and FOXP1-IT1 was co-expressed with HEPACAM and ST6GALNAC1. Moreover, FOXP1-IT1 was a protective factor while LINC01560 was a risky factor for COAD recurrence. Higher expression of LINC01560 was related to poorer survival, whereas upregulation of FOXP1-IT1 exhibited good survival. LINC01560 was reported to be aberrantly expressed in osteosarcoma [34]. CXCL14, termed breast and kidney expressed chemokine (BRAK), is one conserved chemokine involved in the activation of various immune cells such as natural killer cells, immature dendritic cells, and macrophages [35]. Consistent with our study, the report of Zeng et al. has found the significantly elevated expression level of CXCL14 in colorectal carcinoma tissue compared with normal tissues, and 


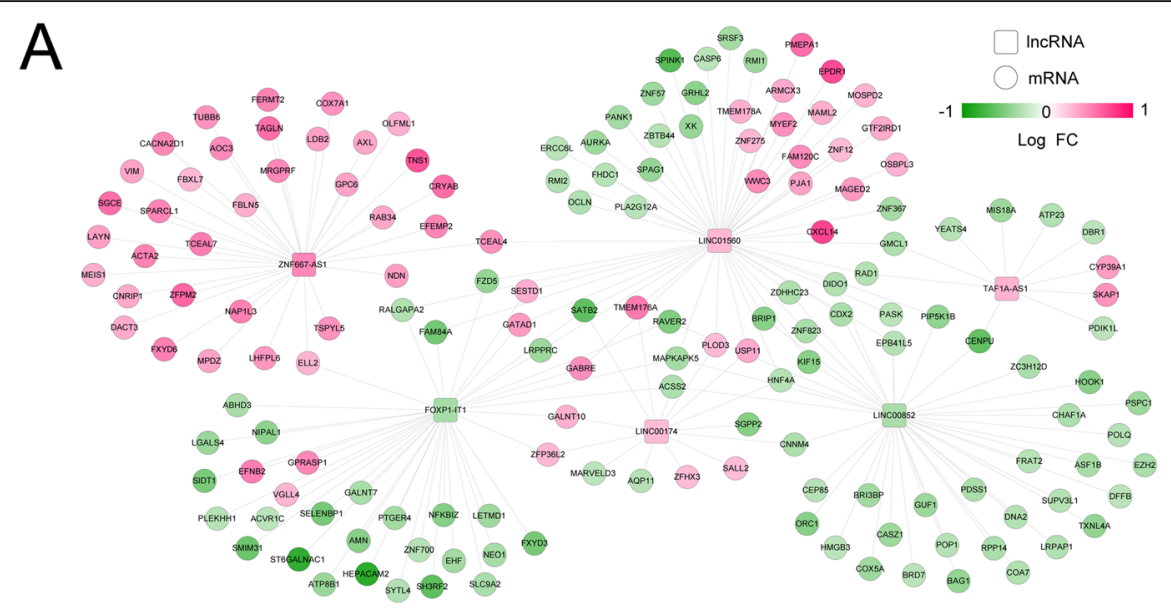

B

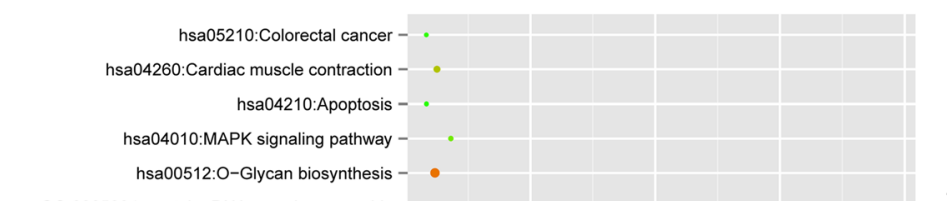
GO:0065004 protein-DNA complex assembly GO:0051252 regulation of RNA metabolic process GO:0045449 regulation of transcription GO:0034728 nucleosome organization GO:0034284 response to monosaccharide stimulus $\stackrel{E}{\frac{E}{L}}$ GO:0032355 response to estradiol stimulus GO:0031497 chromatin assembly GO:0009749 response to glucose stimulus GO:0009746 response to hexose stimulus -

GO:0009743 response to carbohydrate stimulus GO:0007164 establishment of tissue polarity GO:0007017 microtubule-based process GO:0006355 regulation of transcription, DNA-dependent GO:0006350 transcription GO:0006334 nucleosome assembly GO:0006323 DNA packaging GO:0006260 DNA replication -

$$
\begin{aligned}
& -\log (\text { PValue, 10) } \\
& -\quad 1.5 \\
& -\quad 2.0 \\
& -\quad 2.5 \\
& -\quad 3.0 \\
& -\log (\text { PValue, 10) } \\
& -3.0 \\
& -2.5 \\
& -2.0 \\
& -1.5
\end{aligned}
$$

5

10

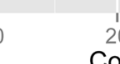

Fig. 6 Construction of co-expression network and functional analysis. a The co-expression network of differentially expressed (DE) IncRNAs and DEmRNAs. The red color shows the upregulated IncRNAs or mRNAs, and the green color denotes the downregulated IncRNAs or mRNAs. Squares and circles, respectively, represent IncRNAs and mRNAs. b GO and KEGG pathways of DEmRNAs. Horizontal axis and vertical axis represent the gene number and term, respectively; the color and size of the bots indicate the significant $P$ value, and the closer the color is to red, the higher the significance

high CXCL14 expression increased the recurrence risk of colorectal carcinoma [36]. EPDR1, which encodes for type II transmembrane protein, is originally identified in teleost fishes and involved in cell adhesion [37]. Gimeno et al. have revealed upregulated EPDR1 in colorectal carcinoma tissue, and EPDR 1 may be a potential biomarker of tumor invasiveness in patients with colorectal carcinoma [38]. However, whether IncRNA-CXCL14/EPDR1 axis was associated with the survival of patients with COAD recurrence still needs to be investigated in the following analysis.
HEPACAM is originally discovered in the liver and then considered as one member of the immunoglobulin superfamily [39]. Recent studies have demonstrated HEPACAM was under-expressed in several cancers. A recent study has shown that $H E P A C A M$ was expressed at low level, and HEPACAM overexpression inhibited cell proliferation, migration, and invasion in colorectal cancer [40]. ST6GALNAC1 is a key sialyltransferase for the biosynthesis of the cancer-associated Sialyl-Tn (STn) antigen that involved in cell adhesion, invasion, and metastasis in cancers [41]. Several studies have suggested that ST6GalNAc1 is overexpressed in breast and colon 
cancer, and upregulation of ST6GalNAc1 promotes tumor growth and metastasis [42, 43]. Unfortunately, the relationships between FOXP1-IT1-HEPACAM/ ST6GALNAC1 and prognosis of patients with COAD recurrence have not been elucidated.

We found that ZNF667-AS1 was another risky factor for survival evaluation of patients with COAD. Previous studies have demonstrated that ZNF667-AS1 was dysregulated in multiple cancers and associated with tumor invasion and metastasis [44-46]. Peng et al. recurrently constructed a IncRNA-related competing endogenous RNA network and highlighted that ZNF667-AS1 was a predictor for survival prognosis of gastric cancer [47]. Several researchers also suggested that LINC00174 played significant roles in the molecular pathogenesis of several cancers, including hepatocellular carcinoma and colorectal carcinoma $[48,49]$. Our analysis revealed that the overexpression of ZNF667-AS1 was related to the poor prognosis of patients with COAD recurrence. However, the influences of ZNF667-AS1, LINC00174, LINC00852, and TAF1A-AS1 on COAD recurrence have not been completely illuminated. Therefore, we will collect more clinical information to explore the effects of combination of these lncRNA signatures on COAD. Moreover, corresponding function mechanisms were also required to investigate by in vitro and in vivo assays LINC00852, LINC01560, and LINC00174.

\section{Conclusion}

In conclusion, this study constructed a SVM classifier for identifying recurrent COAD patients and prognostic prediction model for COAD recurrence. To our knowledge, this is the first study to screen six lncRNA signatures for predicting COAD recurrence, which is important to develop a novel therapeutic strategy for improving the survival of patients with COAD recurrence. However, the underlying mechanism of these lncRNA signatures should be further investigated.

\section{Abbreviations}

COAD: Colon adenocarcinoma; IncRNAs: Long non-coding RNAs: NCBI: National Center for Biotechnology Information; TCGA: The Cancer Genome Atlas; RFE: Recursive feature elimination; SVM: Algorithm based on the support vector machine; AUC: Area under the curve; ROC: Receiver operating characteristic; Sen: Sensitivity; Spe: Specificity; PPV: Positive prediction value; NPV: Negative prediction value; RS: Risk score; K-M: KaplanMeier; DERs: Differentially expressed RNAs; DAVID: Database for Annotation, Visualization, and Integrated Discovery; GO: Gene Ontology; KEGG: Kyoto Encyclopedia of Genes and Genomes

\section{Authors' contributions}

Lei Wang and Lipeng Jin conceived, designed, and performed the experiments. Chenyao Li and Tao Liu analyzed and interpreted the data. Lipeng Jin contributed to the methods, materials, and analysis tools or data. Lei Wang wrote the paper. All authors read and approved the final manuscript.

\section{Funding}

The authors received no financial support for this study.
Availability of data and materials

The data and materials are available under the permission of author.

Ethics approval and consent to participate

Not applicable.

\section{Consent for publication}

All authors of this paper consent for publishing the manuscript and figures in the journal.

\section{Competing interests}

The authors declare that they have no competing interests.

Received: 9 October 2019 Accepted: 13 May 2020

Published online: 10 June 2020

\section{References}

1. Siegel RL, Miller KD, Jemal A. Cancer statistics, 2019. CA: a cancer journal for clinicians. 2019;69(1):7-34.

2. Hashiguchi $Y$, Muro K, Saito $Y$, Ito $Y$, Ajioka Y, Hamaguchi T, Hasegawa K, Hotta K, Ishida H, Ishiguro M. Japanese Society for Cancer of the Colon and Rectum (JSCCR) guidelines 2019 for the treatment of colorectal cancer. International journal of clinical oncology. 2019:1-42.

3. Vatandoost N, Ghanbari J, Mojaver M, Avan A, Ghayour-Mobarhan M, Nedaeinia R, Salehi R. Early detection of colorectal cancer: from conventional methods to novel biomarkers. Journal of cancer research and clinical oncology. 2016;142(2):341-51.

4. Sanoff HK, Sargent DJ, Campbell ME, Morton RF, Fuchs CS, Ramanathan RK, Williamson SK, Findlay BP, Pitot HC, Goldberg RM. Five-year data and prognostic factor analysis of oxaliplatin and irinotecan combinations for advanced colorectal cancer: N9741. Journal of Clinical Oncology. 2008;26(35): 5721.

5. Wardle J, Robb K, Vernon S, Waller J. Screening for prevention and early diagnosis of cancer. American psychologist. 2015;70(2):119.

6. Anastasiadou E, Jacob LS, Slack FJ. Non-coding RNA networks in cancer. Nature Reviews Cancer. 2018;18(1):5.

7. Iyer MK, Niknafs YS, Malik R, Singhal U, Sahu A, Hosono Y, Barrette TR, Prensner JR, Evans JR, Zhao S. The landscape of long noncoding RNAs in the human transcriptome. Nature genetics. 2015:47(3):199.

8. Bhan A, Soleimani M, Mandal SS. Long noncoding RNA and cancer: a new paradigm. Cancer research. 2017;77(15):3965-81.

9. Zhou M, Guo M, He D, Wang X, Cui Y, Yang H, Hao D, Sun J. A potential signature of eight long non-coding RNAs predicts survival in patients with non-small cell lung cancer. Journal of translational medicine. 2015;13(1):231.

10. Zhou M, Sun Y, Sun Y, Xu W, Zhang Z, Zhao H, Zhong Z, Sun J. Comprehensive analysis of IncRNA expression profiles reveals a novel IncRNA signature to discriminate nonequivalent outcomes in patients with ovarian cancer. Oncotarget. 2016;7(22):32433.

11. Zhou M, Zhao H, Wang Z, Cheng L, Yang L, Shi H, Yang H, Sun J. Identification and validation of potential prognostic IncRNA biomarkers for predicting survival in patients with multiple myeloma. Journal of experimental \& clinical cancer research. 2015;34(1):102.

12. Li J, Xue W, Lv J, Han P, Liu Y, Cui B. Identification of potential long noncoding RNA biomarkers associated with the progression of colon cancer. Oncotarget. 2017:8(44):75834.

13. Luo J, Qu J, Wu D-K, Lu Z-L, Sun Y-S, Qu Q. Long non-coding RNAs: a rising biotarget in colorectal cancer. Oncotarget. 2017:8(13):22187.

14. Chen H, Xu J, Hong J, Tang R, Zhang X, Fang J-Y. Long noncoding RNA profiles identify five distinct molecular subtypes of colorectal cancer with clinical relevance. Molecular oncology. 2014;8(8):1393-403.

15. Marisa L, de Reyniès A, Duval A, Selves J, Gaub MP, Vescovo L, EtienneGrimaldi M-C, Schiappa R, Guenot D, Ayadi M. Gene expression classification of colon cancer into molecular subtypes: characterization, validation, and prognostic value. PLoS medicine. 2013;10(5):e1001453.

16. Wright MW. A short guide to long non-coding RNA gene nomenclature. Human genomics. 2014;8(1):7.

17. Ritchie ME, Phipson B, Wu D, Hu Y, Law CW, Shi W, Smyth GK. Limma powers differential expression analyses for RNA-sequencing and microarray studies. Nucleic acids research. 2015;43(7):e47. 
18. Eisen MB, Spellman PT, Brown PO, Botstein D. Cluster analysis and display of genome-wide expression patterns. Proceedings of the National Academy of Sciences. 1998;95(25):14863-8.

19. Wang L, Cao C, Ma Q, Zeng Q, Wang H, Cheng Z, Zhu G, Qi J, Ma H, Nian H. RNA-seq analyses of multiple meristems of soybean: novel and alternative transcripts, evolutionary and functional implications. BMC plant biology. 2014;14(1):169

20. Wang Q, Liu X. Screening of feature genes in distinguishing different types of breast cancer using support vector machine. OncoTargets and therapy. 2015;8:2311.

21. Deist TM, Dankers FJ, Valdes G, Wijsman R, Hsu IC, Oberije C, Lustberg T, van Soest J, Hoebers F, Jochems A. Machine learning algorithms for outcome prediction in (chemo) radiotherapy: an empirical comparison of classifiers. Medical physics. 2018;45(7):3449-59.

22. Robin X, Turck N, Hainard A, Tiberti N, Lisacek F, Sanchez J-C, Müller M. pROC: an open-source package for R and S+ to analyze and compare ROC curves. BMC bioinformatics. 2011;12(1):77.

23. Wang $P$, Wang $Y$, Hang B, Zou X, Mao J-H. A novel gene expression-based prognostic scoring system to predict survival in gastric cancer. Oncotarget. 2016;7(34):55343

24. Mayr A, Schmid M. Boosting the concordance index for survival data-a unified framework to derive and evaluate biomarker combinations. PloS one. 2014;9(1):e84483.

25. Zhang X, Li Y, Akinyemiju T, Ojesina Al, Buckhaults P, Liu N, Xu B, Yi N. Pathway-structured predictive model for cancer survival prediction: a twostage approach. Genetics. 2017;205(1):89-100.

26. Schröder MS, Culhane AC, Quackenbush J. Haibe-Kains B: survcomp: an R/ Bioconductor package for performance assessment and comparison of survival models. Bioinformatics. 2011;27(22):3206-8.

27. Zou KH, Tuncali K, Silverman SG. Correlation and simple linear regression. Radiology. 2003;227(3):617-28.

28. Huang DW, Sherman BT, Lempicki RA. Systematic and integrative analysis of large gene lists using DAVID bioinformatics resources. Nature protocols. 2009:4(1):44.

29. Huang DW, Sherman BT, Lempicki RA. Bioinformatics enrichment tools: paths toward the comprehensive functional analysis of large gene lists. Nucleic acids research. 2008;37(1):1-13.

30. Rodríguez-Ezpeleta N, Hackenberg M, Aransay AM. Bioinformatics for high throughput sequencing. Springer Science \& Business Media. 2011.

31. Huang S, Cai N, Pacheco PP, Narrandes S, Wang Y, Xu W. Applications of support vector machine (SVM) learning in cancer genomics. Cancer Genomics-Proteomics. 2018;15(1):41-51.

32. Feres M, Louzoun Y, Haber S, Faveri M, Figueiredo LC, Levin L. Support vector machine-based differentiation between aggressive and chronic periodontitis using microbial profiles. International dental journal. 2018;68(1):39-46.

33. Bao Z, Zhang W, Dong D. A potential prognostic IncRNA signature for predicting survival in patients with bladder urothelial carcinoma. Oncotarget. 2017:8(6):10485.

34. Jiang M, Wang YR, Xu N, Zhou L, An Q. Long noncoding RNA MEG3 play an important role in osteosarcoma development through sponging microRNAs. J Cell Biochem. 2019;120(4):5151-9.

35. Lu J, Chatterjee M, Schmid H, Beck S, Gawaz M. CXCL14 as an emerging immune and inflammatory modulator. Journal of Inflammation. 2016;13(1):1.

36. Zeng J, Yang X, Cheng L, Liu R, Lei Y, Dong D, Li F, Lau QC, Deng L, Nice EC. Chemokine CXCL14 is associated with prognosis in patients with colorectal carcinoma after curative resection. Journal of translational medicine. 2013;11(1):6.

37. Shashoua VE. The role of brain extracellular proteins in neuroplasticity and learning. Cellular and molecular neurobiology. 1985;5(1):183-207.

38. Gimeno-Valiente F, Riffo-Campos A, Tarazona N, Montón-Bueno J, Gambardella V, Huerta M, Cervantes A, López-Rodas G, Franco L, Castillo J. PO-182 The upregulation of EPDR1 is related to tumour invasiveness in a cohort of localised colorectal cancer patients. In.: BMJ Publishing Group Limited. 2018.

39. Lee LH, Moh MC, Shen S. A novel Ig-like cell adhesion molecule hepaCAM, frequently lost in diverse human cancers, promotes cell-matrix interactions and inhibits cell growth. In.: AACR. 2007.

40. H-t G, R-j C, Cheng L, Liu C-Y. Overexpression of hepatocyte cell adhesion molecule (hepaCAM) inhibits the proliferation, migration, and invasion in colorectal cancer cells. Oncology Research Featuring Preclinical and Clinical Cancer Therapeutics. 2017;25(7):1039-46.
41. Ikehara Y, Kojima N, Kurosawa N, Kudo T, Kono M, Nishihara S, Issiki S, Morozumi K, Itzkowitz S, Tsuda T. Cloning and expression of a human gene encoding an $\mathrm{N}$-acetylgalactosamine-a2, 6-sialyltransferase (ST6GalNAC I): a candidate for synthesis of cancer-associated sialyl-Tn antigens. Glycobiology. 1999;9(11):1213-24.

42. Julien S, Adriaenssens E, Ottenberg K, Furlan A, Courtand G, VercoutterEdouart A-S, Hanisch F-G, Delannoy P, Le Bourhis X. ST6GalNAc I expression in MDA-MB-231 breast cancer cells greatly modifies their O-glycosylation pattern and enhances their tumourigenicity. Glycobiology. 2005;16(1):54-64.

43. Ozaki H, Matsuzaki $H$, Ando $H$, Kaji $H$, Nakanishi $H$, Ikehara $Y$, Narimatsu $H$. Enhancement of metastatic ability by ectopic expression of ST6GalNAcl on a gastric cancer cell line in a mouse model. Clinical \& experimental metastasis. 2012;29(3):229-38.

44. Li YJYZ, Wang YY, Wang Y. Long noncoding RNA ZNF667-AS1 reduces tumor invasion and metastasis in cervical cancer by counteracting microRNA-93-3p-dependent PEG3 downregulation. Mol Oncol. 2019;13(11): 2375-92.

45. Dong ZLS, Wu X, Niu Y, Liang X, Yang L, Guo Y, Shen S, Liang J, Guo W. Aberrant hypermethylation-mediated downregulation of antisense IncRNA ZNF667-AS1 and its sense gene ZNF667 correlate with progression and prognosis of esophageal squamous cell carcinoma. Dong Z, Li S, WU X, Niu Y, Liang X, Yang L, Guo Y, Shen S, Liang J, Guo W. 2019;10(12):930.

46. Meng WCW, Zhao L, Chi W, Cao H, Wang B. Aberrant methylation and downregulation of ZNF667-AS1 and ZNF667 promote the malignant progression of laryngeal squamous cell carcinoma. J Biomed Sci. 2019;26(1): 13.

47. Peng S, Yin X, Zhang Y, Mi W, Li T, Yu Y, Jiang J, Liu Q, Fu Y. Competing endogenous RNA network analysis reveals potential long non-coding RNAs as predictive biomarkers of gastric cancer. Oncol Lett. 2020;19(3):2185-96.

48. Zhao JTCB, Sun Y, Chi NN, Zhang XM, Sun JB, Chen Y, Xia Y. LINC00174 is an oncogenic IncRNA of hepatocellular carcinoma and regulates miR-320/ S100A10 axis. Cell Biochem Funct. 2020.

49. Shen YGX, Tan W, XU T. STAT1-mediated upregulation of IncRNA LINC00174 functions a ceRNA for miR-1910-3p to facilitate colorectal carcinoma progression through regulation of TAZ. Gene. 2018;666:64-71.

\section{Publisher's Note}

Springer Nature remains neutral with regard to jurisdictional claims in published maps and institutional affiliations.

Ready to submit your research? Choose BMC and benefit from:

- fast, convenient online submission

- thorough peer review by experienced researchers in your field

- rapid publication on acceptance

- support for research data, including large and complex data types

- gold Open Access which fosters wider collaboration and increased citations

- maximum visibility for your research: over $100 \mathrm{M}$ website views per year

At BMC, research is always in progress.

Learn more biomedcentral.com/submissions 